\title{
L'analisi economica delle relazioni tra ICT e organizzazioni sanitarie
}

\begin{abstract}
Riassunto
Questo lavoro, partendo dall'approccio teorico neoistituzionalista, analizza gli effetti dei crescenti investimenti in ICT sui confini delle imprese, con particolare riferimento alla dimensione dell'integrazione verticale. L'analisi dell'impatto delle ICT sulle organizzazioni sanitarie fa emergere un effetto assai diverso dall'accorciamento dei legami verticali tra varie fasi della produzione che si osserva tendenzialmente in altri ambiti. Infatti, la crescente evoluzione dei servizi sanitari ad organizzarsi quali insieme di attività e servizi eterogenei ma fortemente integrati e finalizzati alla prevenzione, al mantenimento e al recupero del migliore stato di salute possibile, amplifica i vantaggi comparati derivanti da una gestione integrata anche dei flussi informativi che, a sua volta, rafforza i vantaggi di soluzioni organizzative verticalmente integrate.

Parole chiave - Costi di transazione, modelli organizzativi, servizi sanitari, ICT, gestione integrata, valutazione economica
\end{abstract}

Abstract (The economic analysis of the relationship between ICT and health organizations)

Starting from the neo-institutional theoretical approach, this article analyses the effects of the growing ICT investments on the boundaries of economic organizations, with particular reference to the dimension of vertical integration. The analysis of the impact of ICT on health organizations reveals very different effects than the shortening of vertical links between various stages of production that is typically observed in other industries. Indeed, the growing trend of health systems to strengthen the institutional and managerial tools to integrate their heterogeneous activities and services in order to provide a more effective support to the prevention, maintenance and recovery of the highest attainable standard of health, enhances the comparative advantages arising from an integrated management of information flows, which further strengthens the advantages of vertically integrated organizational solutions.

Key words- Transaction costs, organizational models, health care services, ICT, integrated care, economic evaluation 


\section{Introduzione}

Il lavoro si propone di riflettere sul contributo dell'analisi economica delle istituzioni alla comprensione delle relazioni tra organizzazione dei sistemi sanitari e crescente impiego di Information and Communication Technologies (ICT) sia per lo svolgimento di funzioni amministrative e gestionali sia per funzioni direttamente connesse ai processi assistenziali. A questo scopo, la sezione 2 illustra brevemente le linee di fondo della teoria dell'impresa ispirata dal contributo di Coase (1937), come strumento organizzativo per contenere i costi di transazione i cui confini dipendono da valutazioni di vantaggio relativo tra transazioni gestite mediante contratto o assetto gerarchico. La sezione 3 è dedicata a esplicitare le implicazioni di questo approccio teorico alle organizzazioni che erogano servizi sanitari in presenza di un finanziatore unico, confrontando i vantaggi comparati di modelli verticalmente integrati rispetto a quelli che si affidano maggiormente a transazioni di mercato. In questo ambito, sono esaminate le ragioni della recente tendenza a privilegiare l'adozione di assetti integrati che meglio si adattano ai più recenti sviluppi demografici dal lato della domanda e scientifico-tecnologici dal lato dell'offerta, in particolare per la loro capacità di contenere $\mathrm{i}$ costi di transazione per la gestione di percorsi assistenziali.

La sezione 4 analizza gli effetti della recente accelerazione nella diffusione di ICT in grado di potenziare i flussi informativi a disposizione di produttori e consumatori e quindi di modificare in modo strutturale le determinanti dei confini delle imprese. Infatti, nella generalità dei mercati, le imprese possono ora valutare l'acquisizione di fattori produttivi da parte di soggetti esterni utilizzando in modo sistematico e a costi pressoché nulli la possibilità di confrontare ex ante e di verificare ex post le condizioni contrattuali. In questo modo viene enfatizzato uno dei principali ritorni dagli investimenti in ICT dato dalla loro capacità di ridurre i costi di transazione delle imprese nell'acquisizione di beni e servizi al di fuori dei propri confini mediante una riduzione delle asimmetrie informative nei confronti di comportamenti potenzialmente opportunistici di fornitori esterni che agiscono in condizioni di azzardo morale. La sezione 5 affronta invece le ragioni per cui questi effetti hanno una rilevanza minore nel caso dei servizi sanitari, che si stanno sempre più strutturando in percorsi assistenziali integrati con un forte coordinamento tra più erogatori capace di fronteggiare meglio la complessità delle informazioni rilevanti per le scelte cliniche e gestionali. In questo ambito, è probabile che il potenziamento delle possibilità di raccolta, gestione e analisi dei dati 
relativi allo stato di salute degli assistiti e alle caratteristiche e agli effetti dei trattamenti offerti contribuisca a rafforzare i vantaggi comparati delle organizzazioni verticalmente integrate che possono disporre della titolarità delle informazioni relative all'insieme degli interventi realizzati e dei loro esiti nelle diverse fasi dei percorsi assistenziali senza costi di transazione aggiuntivi.

In sezione 6 il lavoro si concentra invece sui problemi della valutazione economica degli effetti dell'adozione di ICT in ambito sanitario sia a supporto dei processi amministrativi e gestionali - per lo più rivolte a superare i problemi di interoperabilità tra i diversi sistemi informativi utilizzati -, sia dei processi assistenziali che riguardano invece lo sviluppo di dispositivi per la raccolta di dati sullo stato di salute degli assistiti, l'impiego di applicativi per l'analisi di flussi di dati clinici provenienti da fonti diverse e l'impiego dei big data per la valutazione dell'efficacia dei trattamenti, di controllo sui comportamenti di professionisti e strutture nonché di previsione dei fabbisogni e di definizione di strategie di programmazione. L'analisi empirica degli effetti degli investimenti in ICT ha messo in luce non solo i tempi relativamente lunghi perché $i$ benefici delle innovazioni superino i loro costi, ma soprattutto il ruolo degli ostacoli che riducono l'impatto delle nuove tecnologie sui sistemi nel loro complesso e che hanno prevalentemente a che fare con la gestione del capitale umano dei professionisti coinvolti in profondi cambiamenti strutturali nei modelli organizzativi e nella pratica clinica. Sotto questo profilo, si sottolinea l'importanza che la definizione delle politiche pubbliche in tema di sostegno delle ICT, parta anche da una valutazione consapevole degli impatti allocativo e redistributivo sulle posizioni dei diversi gruppi professionali coinvolti, in modo da evitare che i vantaggi potenziali delle innovazioni trovino ostacoli superabili al dispiegamento dei loro effetti. La sezione 7, partendo da questa considerazione analizza in maggior dettaglio alcune innovazioni in ICT che possono rivestire una rilevanza strategica per $i$ responsabili del finanziamento e dell'organizzazione di sistemi sanitari per affinare le funzioni di programmazione, di valutazione di efficacia di trattamenti e modelli assistenziali, nonché per definire soluzioni organizzative e finanziarie più coerenti con gli obiettivi di fondo dei sistemi. La sezione 8 conclude il lavoro con una sintesi dei principali risultati.

\section{Costi di transazione e modelli organizzativi}


La teoria dei costi di transazione fa riferimento a un filone di pensiero sviluppatosi a partire dagli anni settanta e denominato Neoistituzionalismo (New institutional economics), che studia il contesto entro il quale le organizzazioni operano concentrandosi sul ruolo delle istituzioni nell'influenzare il livello di efficienza delle imprese e dei sistemi economici. La teoria dei costi di transazione ha un antecedente importante in un saggio del 1937 di Ronald Coase, premio Nobel per l'economia nel 1991, intitolato The Nature of the Firm in cui Coase propone una teoria dell'impresa basata sul confronto tra costo d'uso del mercato e costo d'uso interno per il governo delle transazioni economiche. Secondo Coase, le imprese sono strutture di governo di un insieme di transazioni che vengono realizzate al proprio interno a costi inferiori a quelli realizzabili sul mercato perché entro i confini delle imprese vigono relazioni gerarchiche che consentono di ridurre $\mathrm{i}$ costi di negoziazione e di verifica rispetto alle soluzioni contrattuali, soprattutto in condizioni di informazione incompleta e imperfetta. Questa minore incidenza nei costi di gestione delle transazioni si verifica sia ex ante (ricerca e analisi delle condizioni proposte dai fornitori potenziali) sia ex post (misurazione e controllo delle prestazioni effettivamente rese). Riducendo questi costi, le imprese si caratterizzano come sistemi di programmazione alternativi e superiori al mercato in termini di efficienza allocativa. Si ha quindi uno spostamento nella concettualizzazione dei tratti essenziali dell'impresa da quelli tecnologicologistici a quelli istituzionali che sottolineano la rilevanza delle sue caratteristiche di struttura stabile di governo delle transazioni tra i titolari dei diritti sui fattori produttivi complementari. A partire da queste caratteristiche si comprende che solo se il coordinamento tra questi titolari di diritti è realizzato in modo efficiente, si metteranno in atto investimenti specifici e irreversibili che a loro volta consentiranno la realizzazione di vantaggi della specializzazione e/o di economie di scala e di varietà. In questo senso, la riduzione dei costi di negoziazione, coordinamento e applicazione dei contratti dovuta al controllo su comportamenti opportunistici da parte di chi conferisce $\mathrm{i}$ fattori nonché la possibilità di allocare i rischi sui soggetti (tipicamente i titolari dei diritti di proprietà sul capitale di rischio) costituiscono i vantaggi comparati di operare all'interno di un assetto istituzionale unitario in cui l'allocazione delle risorse avviene sulla base di regole di programmazione e controllo. ${ }^{1}$

1 Un ulteriore rafforzamento alla scelta di una soluzione integrata viene, sempre all'interno dell'approccio istituzionale e partendo proprio dai presupposti della teoria dei costi di transazione, dalla teoria dei diritti di proprietà che ha origine dai lavori di Grossman 
Trent'anni dopo, dalla metà-fine anni sessanta, Williamson (1975) riprende le intuizioni di Coase e si propone di rendere la teoria utilizzabile anche a fini normativi per definire la struttura organizzativa e le forme di governo ottimali delle imprese a seconda degli ambiti in cui si trovano ad operare. In questa prospettiva ogni organizzazione nasce dal tentativo di minimizzare i costi di transazione in contesti caratterizzati da contratti incompleti, investimenti specifici e razionalità limitata e il compito dell'analisi istituzionale comparata diviene quello di individuare in modo più allargato i meccanismi istituzionali di governo in grado di minimizzare questi costi. In particolare, lo spettro dei meccanismi di governo delle transazioni su cui si concentra l'analisi è inizialmente polarizzato sulle alternative del mercato e della gerarchia che costituiscono le forme di governo alternative di gestione delle attività economiche. Successivamente, l'approccio neo-istituzionalista approfondisce anche l'analisi di strutture di governo intermedie, che emergono quando i rapporti tra più imprese si consolidano nel tempo attraverso la definizione di contratti relazionali di lungo periodo definendo forme organizzative ibride di quasi-integrazione o vere e proprie reti integrate di imprese (Blois 1972; Dietrich 1994). Secondo questi sviluppi, in talune condizioni - definite dalla tecnologia e dalla domanda - le imprese possono fruire di gran parte dei vantaggi dell'integrazione, senza assumere i rischi o le rigidità derivanti da un'unica proprietà. La scelta di una soluzione integrata o quasi-integrata dipende comunque da una pluralità di fattori e in particolare dalla rilevanza attribuita al pieno controllo sulle caratteristiche dei beni oggetto di transazione o alla presenza di investimenti irreversibili, condizioni che favoriscono l'adozione di soluzioni integrate, ovvero dall'importanza di mantenere flessibilità a fronte di possibili modifiche nelle condizioni della domanda o della tecnologia, elemento che aumenta il vantaggio comparato di soluzioni contrattuali. È importante sottolineare che le forme organizzative integrate verticalmente non sono dominanti in tutti i contesti o periodo, ma portano con loro fonti potenziali di inefficienza, soprattutto in un'ottica dinamica che tenga conto della capacità di adattarsi a scenari esterni in rapida evoluzione che impongono una ridefinizione continua dei processi di produzione e dei sistemi di relazione con le altre imprese e $\mathrm{i}$ consumatori finali. La stessa definizione dei confini dell'impresa dipende -

\footnotetext{
e Hart (1986) e Hart e Moore (1990). Per la teoria dei diritti di proprietà la soluzione integrata consente un miglioramento paretiano rispetto a relazioni non integrate in presenza di investimenti specifici il cui valore sia superiore all'interno di quella determinata relazione rispetto a possibili impieghi alternativi.
} 
oltre che dalle caratteristiche degli investimenti caratterizzanti i processi produttivi - dalle informazioni a disposizione dei diversi soggetti e in particolare dalla completezza delle informazioni relative alla tecnologia e alla domanda nello status quo e nelle previsioni sul futuro, dalle caratteristiche delle asimmetrie informative esistenti tra soggetti che conferiscono diversi fattori o tra chi opera dal lato della domanda e dell'offerta.

\section{Costi di transazione e organizzazione dei servizi sanitari}

Se si applica l'approccio dei costi di transazione all'analisi delle organizzazioni attive nell'ambito dei servizi sanitari, si rileva la presenza di molte caratteristiche che raccomanderebbero l'adozione di strutture verticalmente integrate: elevati investimenti specifici e irreversibili a partire da quelli nel capitale umano dei professionisti, complessità dei processi da coordinare sia per la varietà dei fattori produttivi coinvolti sia perché gli esiti dipendono da fattori ampiamente fuori dal controllo di chi eroga $\mathrm{i}$ servizi, elevati costi di verifica nei confronti di possibili comportamenti opportunistici di assistiti e professionisti a causa di profonde e ineliminabili asimmetrie informative e difficoltà nel valutare ex ante ma anche ex post gli esiti dei diversi modelli organizzativi. A fronte di questi elementi, la presenza di un soggetto che ha titolo per impiegare strumenti di programmazione e di coordinamento senza negoziare ogni prestazione con $\mathrm{i}$ singoli titolari dei diritti sui fattori produttivi, sembra garantire una riduzione dei costi di transazione che a sua volta consente una maggiore continuità nei percorsi assistenziali, una superiore capacità di sfruttare economie di scala e di varietà e di sostenere il rischio derivante da pagamenti a quota capitaria. Più specificamente, per quanto concerne la gestione del personale, la presenza di un unico titolare delle funzioni di programmazione che è anche l'unico (o comunque di gran lunga il principale) acquirente di prestazioni professionali consente un più efficace governo degli incentivi dei diversi gruppi professionali, promuovendo forme di collaborazione interdisciplinare e un più rapido adeguamento ai cambiamenti nei percorsi assistenziali che si rendono necessari per soddisfare al meglio i mutevoli fabbisogni degli assistiti. ${ }^{2}$

\footnotetext{
${ }^{2}$ In questo senso, le condizioni che rendono vantaggiosa una scelta di integrazione non sono logicamente diverse da quelle che favoriscono la definizione di contratti relazionali di lungo periodo per contenere possibili comportamenti opportunistici e per definire le
} 
Nonostante il fatto che i sistemi sanitari presentino molte delle caratteristiche che attribuiscono un vantaggio comparato agli assetti di governo di tipo verticalmente integrato, negli anni novanta, sotto la spinta del nuovo paradigma di gestione del settore pubblico denominato New Public Management, in alcuni paesi sono stati introdotti assetti istituzionali tali da separare i finanziatori dagli erogatori dei servizi, definire i rapporti tra questi su base contrattuale e incentivare forme di concorrenza tra $\mathrm{i}$ secondi resi autonomi da interventi normativi ad hoc di dis-integrazione verticale ("quasi-mercati" nella definizione di Wiliamson (1975), poi ripresa da Enthoven (1988) e Le Grand (2002)). Queste riforme hanno ricevuto molta attenzione soprattutto nel caso della Gran Bretagna, ${ }^{3}$ ma per lo più sono state poi seguite da interventi di segno opposto dovuti alla presa d'atto che i rapporti contrattuali instaurati tra finanziatori ed erogatori, orientati necessariamente al lungo periodo per la presenza di ingenti investimenti irreversibili e per la necessità di garantire forme di stringente coordinamento tra gli erogatori in funzione di obiettivi di continuità di cura, avevano portato rapidamente al ripristinarsi di quelle forme (implicite) di integrazione verticale che ci si proponeva di eliminare. ${ }^{4}$ Queste esperienze hanno mostrato che le alternative percorribili per introdurre elementi concorrenziali in un contesto in cui si mantiene un finanziatore unico sono due: o si adotta un modello di competizione - anche tra erogatori pubblici completamente orientato dalle scelte degli assistiti (France, 1998), oppure

condizioni per effettuare investimenti specifici. Non è raro infatti che in questo settore $\mathrm{i}$ contratti relazionali tra due soggetti si estendano alla definizione di vere e proprie reti di imprese (integrazione "virtuale") legate tra loro da intensi rapporti di collaborazione. Tali reti, possono essere considerate collusive in un sistema sanitario a carattere privatistico (se sorgono anche con l'intento di limitare la concorrenza), ma in altri contesti possono divenire strumenti organizzativi adatti a sistemi sanitari a finanziatore unico che definisce con erogatori dotati di autonomia imprenditoriali contratti di lungo termine (Ugolini, 2004).

${ }^{3}$ Va sottolineato che questi esperimenti hanno caratteristiche molto diverse rispetto a quelli portati avanti da altri paesi quali l'Olanda o la Germania che hanno sì introdotto assetti concorrenziali, ma tra i soggetti attivi dal lato del finanziamento dei servizi, ai quali poi è stata data la possibilità di definire in autonomia le proprie strategie in merito alla scelta tra forme più o meno accentuate di integrazione o quasi-integrazione con gli erogatori dei servizi.

${ }^{4}$ Il contributo inatteso dei quasi-mercati non è stato tanto nell'introduzione duratura di meccanismi concorrenziali, quando piuttosto nell'aver profondamente modificato il «linguaggio, i valori e l'ambiente istituzionale in cui i servizi vengono prodotti» (Light, 2001), valorizzando l'utilizzo efficiente delle risorse disponibili, la determinazione dei costi effettivi di erogazione delle prestazioni e la valutazione dei loro esiti sulla salute, l'appropriatezza e il governo clinico, elementi che si sono bene integrati anche nei modelli di programmazione negoziata che sono stati via via adottati dopo la breve esperienza dei quasimercati. 
si adotta un meccanismo di concorrenza per il mercato con l'aggiudicazione dei diritti esclusivi a fornire servizi per un dato periodo di tempo. Tuttavia, nel primo caso si espone il finanziatore a gravi rischi di scrematura della domanda, di perdita di coordinamento nella gestione dei percorsi e di sfondamento dei vincoli di bilancio, mentre nel secondo caso vi sono gravi difficoltà a garantire effettiva contendibilità in presenza di affidamenti di durata proporzionale all'entità degli investimenti richiesti e tali per cui, a fronte di comportamenti opportunistici del concessionario, non sarebbe possibile trovare soluzioni alternative in tempi accettabili. In entrambe le opzioni peraltro, se ci si pone qualche obiettivo di minima quanto a qualità e equità nell'accesso alle cure, i decisori pubblici devono potenziare le risorse dedicate alla regolamentazione degli erogatori, il che introduce per altra via, quegli aumenti nei costi di transazione che erano alla base della scelta di assetti organizzativi verticalmente integrati.

Venendo ad anni più recenti, a fronte dell'accelerazione nell'invecchiamento della popolazione e in alcune dinamiche della ricerca scientifica e delle innovazioni tecnologiche, la struttura dell'offerta dei servizi è stata oggetto di una ulteriore e significativa trasformazione. Semplificando, si potrebbe dire che la principale novità riguarda il più accentuato orientamento a definire l'obiettivo dei servizi in termini di assunzione di responsabilità - in parte condivisa con gli assistiti - nel mantenimento del migliore stato di salute possibile. In coerenza a questo approccio, i sistemi sanitari considerano il rapporto con gli assistiti come un processo continuo di interventi, prevalentemente mirati alla formazione, alla vigilanza e alla prevenzione, e con interventi in fase acuta che sono anch'essi per quanto possibile realizzati in modalità che rispettano le loro normali condizioni di vita. Per questo motivo, aumenta la varietà di interventi attuati da professionisti e/o da strutture con compiti altamente differenziati e su tempi molto più lunghi con una crescente importanza delle funzioni di coordinamento e di ponderazione tra incentivi diversi, se non del tutto divergenti, dei vari operatori coinvolti, riducendo il complesso dei costi di transazione e di erogazione degli interventi. Si assiste quindi a una crescente enfasi sulla definizione di percorsi assistenziali disegnati per integrare i diversi apporti dei professionisti e delle strutture orientandoli alla soddisfazione delle esigenze complessive degli assisiti, spesso cronici e con bisogni che valicano il confine con l'assistenza sociale, e con un orientamento più puntuale a ricercare $\mathrm{i}$ modelli organizzativi in grado di conseguire risultati tangibili in termini di salute. Per queste ragioni, in molti paesi ad alto reddito e interessati alle dinamiche demografiche e tecnologiche sopra richiamate, gli interventi di politica sanitaria, così come 
il disegno delle politiche di incentivazione fiscale, sono mirati a favorire lo sviluppo delle organizzazioni che adottano modelli verticalmente integrati. In tali modelli, i finanziatori (integrandosi a valle) o gli erogatori (integrandosi a monte) organizzano i fattori produttivi intorno ai fabbisogni complessivi e di lungo termine degli assistiti cercando di ridurre i costi di transazione complessivi facendo leva su relazioni gerarchiche ovvero definendo relazioni contrattuali di tipo relazionale e quindi comunque orientate al lungo termine. In questo modo i soggetti responsabili del governo dell'integrazione verticale possono governare in modo più efficiente i processi assistenziali nel loro insieme, un obiettivo che assume una maggiore rilevanza a fronte di bisogni più complessi e interdipendenti, e ad aspettative crescenti che impongono maggiori investimenti specifici in tecnologie e l'acquisizione di beni e servizi intermedi (in particolare $\mathrm{i}$ farmaci) a costi sempre più alti.

\section{Effetti delle ICT sui costi di transazione e sui confini delle imprese}

Le ICT possono essere considerate tra le General Purposes Technologies (GPT), ovvero tra le tecnologie utilizzate in modo trasversale in diversi ambiti sociali ed economici (Bresnahan e Trajtenberg, 1995). Le GPT si caratterizzano perché capaci di diffusione capillare, perché soggette esse stesse ad innovazioni incrementali che, nel tempo, ne migliorano la qualità e ne riducono i costi e infine perché capaci di favorire ulteriori innovazioni nei processi produttivi in cui sono impiegate. All'interno di questo gruppo, le ICT sono particolarmente importanti per il loro contributo all'efficienza dei meccanismi di coordinamento organizzativo di altri processi produttivi e quindi l'analisi del loro impatto non può prescindere da una profonda comprensione delle logiche organizzative e delle caratteristiche strutturali degli specifici settori di interesse. ${ }^{5}$

\footnotetext{
${ }^{5}$ Con riferimento ai servizi sanitari, impiegheremo il termine ICT per indicare le innovazioni che consentono una riduzione nei costi e/o un miglioramento nella qualità delle attività di raccolta, accesso o distribuzione, conservazione e analisi dei dati relativi non solo alle caratteristiche dei servizi erogati dai professionisti e dalle strutture di offerta, ma anche alle caratteristiche e allo stato di salute degli assistiti nonché ai risultati individuali e collettivi conseguiti. In concreto, gli investimenti in ICT e i relativi servizi riguardano le infrastrutture di rete, i dispositivi e le attrezzatture per la raccolta e la conservazione dei dati, gli applicativi per la gestione e l'analisi dei dati e in risorse umane dedicate. L'origine di tali innovazioni è da ritrovarsi per lo più all'esterno dell'ambito sanitario, incluse quelle che riguardano le applicazioni dei big data, ma negli ultimi anni si stanno sviluppando applicazioni del tutto peculiari in relazione alle specifiche necessità di gestione e di analisi
} 
Se la caratteristica più saliente degli investimenti in ICT è la loro capacità di ridurre $\mathrm{i}$ costi di transazione in virtù della superiore efficienza nel gestire flussi informativi di dimensioni enormi in tempi limitati ${ }^{6}{ }^{6}$ esse dovrebbero aver modificato in profondità il modo in cui si pone la scelta analizzata nelle sezioni precedenti - tra modelli gerarchici a integrazione verticale e modelli contrattuali. In effetti, la riduzione nei costi di diffusione delle informazioni dovuta all'esplosione di internet, ha abbattuto i costi di promozione da parte delle imprese, anche di dimensioni molto piccole rispetto alla domanda complessiva, che possono quindi far conoscere $\mathrm{i}$ propri prodotti su scala globale costringendo tutti i competitori a seguire. Questi sviluppi, hanno ulteriormente incentivato la crescita di imprese specializzate nella predisposizione di applicativi in grado di ridurre drasticamente $\mathrm{i}$ costi di ricerca delle informazioni rilevanti (costi di transazione del contratto) non solo per i consumatori finali, ma anche e forse soprattutto, per le imprese. In particolare per queste ultime, si apre in via non temporanea, la possibilità di esternalizzare tutte le funzioni non essenziali rispetto alla propria attività e cioè tali da non richiedere investimenti specificamente dedicati, sapendo di poter confrontare in ogni momento i prezzi e la qualità dei beni o servizi acquisiti da terzi sempre a una combinazione di prezzo e qualità vicina alla migliore disponibile a livello mondiale.

In linea generale, questi drastici cambiamenti nei meccanismi di raccolta e diffusione delle informazioni su prezzi e qualità disponibili via internet, hanno effettivamente portato con sé un ridisegno dei modelli organizzativi con una riduzione delle transazioni che si svolgono entro i confini della stessa impresa e con il corrispondente ampliamento di quelle supportate da relazioni contrattuali anche di lungo termine. Questo non significa che tutte le imprese abbiano ridotto la lunghezza della propria filiera verticale, ma piuttosto che, a parità di altre ragioni che giustificano il mantenimento di

delle informazioni da parte degli assistiti, degli erogatori e dei finanziatori di servizi sanitari. L'impatto di tali innovazioni è tale da orientare e accelerare profondi cambiamenti non solo nelle modalità di erogazione di specifici trattamenti clinici, ma anche nei modelli organizzativi e di finanziamento dei servizi.

${ }^{6}$ Con l'uso delle ICT il numero delle possibili interazioni diventa pressoché illimitato e il relativo costo trascurabile (Odlyzko, 2012). In particolare, le tecnologie di coordinamento possono generare tre tipi di effetti (Malone, Yates e Bejamin, 1987) che facilitano i flussi informativi consentendo un uso migliore dell'informazione e una conseguente gestione più efficace dell'incertezza, riducendo di conseguenza, i costi ad essa associati: aumentano il flusso informativo per unità di tempo (effetto di comunicazione), collegano più facilmente $i$ contraenti /agenti, (effetto di integrazione), rendono più efficiente ed efficace il processo di scambio (effetti di brokeraggio). 
modelli organizzativi verticali quali la presenza di investimenti specifici di grandi dimensioni e caratterizzati da alto rischio o la presenza di problemi di certificazione della qualità delle forniture, la tendenza generale è stata $\mathrm{a}$ ridurre l'estensione dei confini verticali delle imprese. In parallelo a questa tendenza, le imprese che gestiscono le piattaforme impiegate da imprese e consumatori per informarsi su beni e servizi hanno invece sperimentato una tendenza all'ampliamento dei confini orizzontali proprio perché il valore delle informazioni fornite ai clienti cresce in maniera esponenziale rispetto sia al numero delle imprese che promuovono i propri beni e servizi sia dei clienti.

\section{Effetti delle ICT sull'organizzazione dei servizi sanitari}

Se dal quadro generale, ci si sposta all'ambito dei servizi sanitari, molti dei processi sopra descritti sembrano trovano puntuale riscontro. Infatti, partendo dal mercato assicurativo, chi cerca copertura assicurativa può confrontare in modo più diretto e completo le condizioni di prezzo e qualità della copertura garantita dai diversi assicuratori, pur in presenza di contratti complessi e condizionati rispetto a molte caratteristiche individuali. D'altra parte, gli assicuratori possono acquisire a costi inferiori informazioni in merito a prezzi, condizioni di accesso e caratteristiche qualitative delle prestazioni offerte da singoli professionisti o strutture di erogazione. In questo senso, gli assicuratori possono, utilizzando i più avanzati strumenti informatici, stipulare a costi relativamente ridotti contratti complessi con una molteplicità di strutture e professionisti garantendosi - a parità di altre condizioni - sia una valutazione comparativa più precisa sia la possibilità di definire contratti più efficienti perché condizionati all'effettiva osservazione di variabili di risultato che la riduzione dei costi misurazione rende possibile. ${ }^{7}$ Come sulla generalità degli altri mercati, la riduzione nei costi di raccolta e analisi delle informazioni relative alle condizioni assicurative e alle caratteristiche e agli esiti delle attività dei professionisti e delle strutture, incentiva l'emergere di mediatori, privati o anche pubblici se vi sono problemi nella definizione delle regole per la diffusione di tali informazione, che riducono ulteriormente i costi di transazione. Sotto questo profilo, si potrebbe quindi ipotizzare che le innovazioni in materia di

\footnotetext{
${ }^{7}$ Argomenti simili sono applicabili anche alla scelta dei singoli professionisti o delle singole strutture da parte di pazienti individuali che per le caratteristiche della propria polizza o del contesto istituzionali in cui operano possono esercitare tale scelta.
} 
ICT abbiano l'effetto univoco di aumentare la mobilità nella scelta degli assicuratori da parte degli assistiti (ove c'è possibilità di scelta) e degli erogatori nonché di contenere la tendenza alla crescita dell'integrazione verticale tra finanziatori ed erogatori descritta in precedenza a fronte delle diverse dinamiche demografiche e tecnologiche in atto.

In realtà, anche se i maggiori investimenti in ICT consentono di raccogliere, condividere e analizzare dati a costi decrescenti, spingendo chi offre servizi a rendere disponibili più informazioni, nel caso specifico dei servizi sanitari i dati relativi alle coperture assicurative e ai singoli interventi assistenziali sono caratterizzati da forme di complessità interpretativa che li rendono del tutto particolari. Infatti, essi tipicamente non sono disponibili in forme che consentono di ricostruire un quadro comparativo tale da chiarire come diversi assicuratori - a parità di premio della polizza - si fanno carico dei fabbisogni di assistiti in condizioni cliniche comparabili e con obiettivi di mantenimento o miglioramento delle condizioni di salute sul lungo termine. Allo stesso modo, anche ponendosi obiettivi di valutazione comparativa meno ambiziosi, come quelli che normalmente si pone un assistito nella scelta tra diverse strutture $o$ professionisti limitatamente a un dato intervento, non è possibile acquisire informazioni attendibili in termini di qualità comparata, dato che è molto difficile rendere i dati davvero confrontabili in considerazione della grande eterogeneità dei singoli casi e visto che gli esiti dei trattamenti sono riferiti a intervalli di tempo relativamente brevi. Non è quindi ovvio che l'attuale disponibilità di informazioni su prezzi ed esiti, pur essendo sicuramente maggiore rispetto al passato, consenta di colmare in modo soddisfacente le asimmetrie informative che sono alla base della scelta di molte organizzazioni del settore di adottare forme organizzative verticalmente integrate.

Inoltre, la riduzione nei costi di raccolta, gestione e analisi dei dati, non è di per sé condizione sufficiente per effettuare investimenti in ICT tali da rendere disponibili le informazioni anche per il modo in cui sono attribuiti i diritti di proprietà e di uso dei dati. Le informazioni, infatti, non sono un bene pubblico in senso economico dato che su di esse è possibile definire chiari diritti di proprietà e di uso, pur essendo invece un bene "non rivale nel consumo" e cioè tale da non presentare costi ulteriori a fronte di un incremento nel numero dei soggetti ammessi al consumo (bene di club). Di norma gli assistiti, possono disporre delle informazioni relative al proprio stato di salute e ai trattamenti loro erogati, ma non hanno incentivi a investire nella loro gestione coordinata a livello di sistema. Allo stesso modo, le strutture di erogazione o i singoli professionisti hanno diritti d'uso 
delle informazioni relative ai loro assistiti rigidamente limitate allo scopo di provvedere al meglio ai bisogni di salute degli assistiti. In alcuni contesti possono diffondere questi dati in forma anonima, ma non sono in grado da soli di costruire banche dati sufficienti per dimensione e dettagli capaci di fornire informazioni effettivamente utili alle decisioni di altre imprese o degli assistiti. Diversa è la posizione degli assicuratori di grandi dimensioni o dei finanziatori nel caso di servizi sanitari pubblici, i quali hanno necessariamente accesso a gran parte delle informazioni rilevanti - almeno ai dati sui consumi sanitari, ma in realtà anche a quelli sulle caratteristiche degli assistiti - perché devono procedere alla copertura dei relativi costi.

Nel momento in cui l'offerta di servizi sanitari assume più marcatamente la configurazione di un insieme integrato di attività volte alla prevenzione, mantenimento e recupero delle migliori condizioni di salute, la possibilità di raccogliere, gestire e analizzare dati su grandi dimensioni relativi alle caratteristiche specifiche di ciascun assistito, a tutti i consumi sanitari e all'evoluzione delle condizioni di salute, consente di ricostruire i processi decisionali in merito ai percorsi seguiti e agli interventi effettuati; inoltre, mediante il confronto con un numero sufficiente di soggetti omogenei, diventa possibile verificare l'efficacia di lungo termine di singoli atti, di interi percorsi e modelli organizzativi. Nell'attuale contesto, se il finanziatore del sistema non è verticalmente integrato (o per controllo diretto o per contratti relazionali), allora deve acquisire un'ampia gamma di prestazioni (diagnostiche, assistenziali territoriali e ospedaliere, di riabilitazione, ecc.) per conto dei propri assicurati stipulando contratti con i singoli erogatori. Nell'ambito di questi contratti dovrebbe negoziare in modo dettagliato l'acquisizione dell'insieme complesso di informazioni di cui sopra, definendo di volta in volta i limiti d'uso e di conservazione delle stesse nonché le responsabilità conseguenti a inadempimenti, garantendo inoltre la piena inter-operabilità dei sistemi adottati per gestirle e trasmetterle. La disponibilità dell'insieme integrato di queste informazioni, è condizione necessaria per verificare se le decisioni cliniche sono state assunte in un'ottica di miglioramento o mantenimento delle condizioni complessive di salute oppure per trarre vantaggio da rendite di posizione $o$ per trasferire costi su altri erogatori contrattualmente responsabili di altri segmenti del percorso assistenziale. La valutazione di queste possibili esternalità - tra le varie fasi di un percorso assistenziale - richiede l'analisi di gruppi di pazienti omogenei di dimensione e durata sufficienti a effettuare confronti metodologicamente robusti e, a sua volta, può consentire di ottenere informazioni utili a indirizzare le scelte dei modelli 
organizzativi, dei processi decisionali e dei trattamenti più efficaci dato il vincolo sulle risorse.

Nel complesso, la possibilità per i finanziatori integrati verticalmente con gran parte degli erogatori di acquisire le informazioni rilevanti senza sopportare i costi di transazione per la negoziazione dei diritti d'uso delle stesse - oltre ai costi di transazione relativi a contratti ordinari - sembra costituire un ulteriore vantaggio comparato il cui rilievo aumenta al crescere della necessità di coordinare l'assistenza e integrare tra loro gli erogatori. Inoltre, questo vantaggio aumenta - in modo più che proporzionale - al crescere delle dimensioni della raccolta dei dati, poiché la possibilità di impiego di questi ultimi per finalità di progettazione e di valutazione dei trattamenti e dei percorsi dipende in modo cruciale dalla numerosità delle osservazioni su assistiti omogenei, il che per molte condizioni cliniche richiede il conseguimento di livelli dimensionali molto rilevanti. Inoltre, il valore dei dati, sempre per le stesse finalità, aumenta considerevolmente se è possibile integrare informazioni relative a tutto il percorso assistenziale e non solo nella fase precedente o successiva a interventi di maggiore complessità, proprio perché è necessario comprendere le implicazioni di lungo termine di percorsi alternativi. Il controllo di banche dati adeguate rispetto a questi profili è quindi condizione per poter progettare, realizzare e valutare i percorsi assistenziali nel loro complesso, riducendo le perdite di efficienza e di efficacia dovute a scelte non appropriate realizzatesi a causa dei conflitti di interesse tra $i$ diversi erogatori e alla mancanza di incentivi dei singoli a tenere in adeguata considerazione le esternalità tra una fase e l'altra dei percorsi. Al contrario, la presenza di un alto numero di finanziatori in competizione tra loro con accesso ai dati su base negoziale e comunque su dimensioni modeste sia per numero di assistiti sia per numero di erogatori, riduce significativamente il valore complessivo dei dati e quindi i benefici di effettuare investimenti in ICT. ${ }^{8}$

\footnotetext{
${ }^{8}$ Peraltro, a prescindere dal modello di attribuzione dei diritti di proprietà e uso su queste informazioni, i grandi cambiamenti nelle applicazioni ICT ai servizi sanitari hanno fatto sì che tutti i principali attori del settore - finanziatori, strutture di erogazione e professionisti - siano pienamente consapevoli del fatto che il valore di una prestazione assistenziale non è più limitata al beneficio diretto in termini di salute a vantaggio di che ne fruisce, ma include anche il valore delle informazioni relative allo status quo ex ante e agli esiti misurati ex post che, opportunamente integrate ed elaborate, forniscono indicazioni essenziali per una molteplicità di ulteriori analisi di costo-efficacia degli interventi effettuati che interessano una platea di soggetti ancora più ampia (centri di ricerca, imprese farmaceutiche, società di consulenza, ecc.).
} 


\section{Investimenti in ICT e loro effetti sull'efficienza dei sistemi servizi sanitari}

La letteratura internazionale ha indagato due principali aree di sviluppo delle ICT applicate all'ambito sanitario, con un'attenzione particolare alla valutazione dei loro effetti sull'efficienza complessiva dei settori a cui sono applicate. In particolare, l'analisi empirica si è concentrata sugli effetti delle ICT in due aree: il supporto ai processi amministrativi e gestionali con l'obiettivo prioritario di unificare e rendere interoperabili i sistemi informativi impiegati da professionisti, imprese o sistemi diversi (CUP, FSE, ricetta dematerializzata, certificati di malattia digitali, ecc.) e il supporto ai processi assistenziali, ambito nel quale particolare interesse hanno destato le applicazioni per il supporto alla telemedicina, ma anche l'impiego di tecniche per l'analisi di big data e di strumenti di intelligenza artificiale per finalità di valutazione e di previsione.

In termini generali, Buntin et al. (2011) mostrano che dei 154 studi esaminati per analizzare gli effetti di innovazioni ICT in ambito sanitario l'analisi costi-benefici si conclude nel $92 \%$ dei casi con risultati positivi. Più specificamente, emergono quattro risultati di particolare interesse: le principali resistenze alla diffusione delle ICT e al dispiegarsi dei loro positivi effetti è imputabile alle difficoltà di coinvolgere pienamente il personale, soprattutto se all'introduzione dell'innovazione non si associa una forte leadership della direzione e azioni specifiche per il coinvolgimento di tutto il personale sanitario (il cosiddetto staff «buy-in»); anche per questa ragione, occorrono tempi lunghi perché i benefici di progetti innovativi superino i costi; vi sono difficoltà di diffusione in ambiti caratterizzati da un'elevata frammentazione nella struttura organizzativa; ${ }^{9}$ l'impatto delle ICT comincia ad essere rilevabile anche nelle organizzazioni di dimensioni minori. ${ }^{10}$

Con riferimento all'importanza di curare la complementarità tra innovazioni ICT e professionisti impegnati nel sistema sanitario e al profilo temporale dei costi e dei benefici, è noto che i primi studi sull'impatto delle ICT avevano evidenziato che i ritorni sull'efficienza dei processi produttivi sono inizialmente limitati (Solow, 1987). Tuttavia, diversi lavori successivi

\footnotetext{
${ }^{9}$ Ad esempio, negli Stati Uniti l'adozione e diffusione dei tracciati record elettronici sono state più lente rispetto alle attese, soprattutto in confronto ad altri settori industriali, mentre più rapida è invece l'adozione da parte dei medici di tecnologie portatili per accedere in remoto ai sistemi informativi disponibili.

${ }^{10}$ Chaudhry et al. (2006) per il periodo 1995-2004; Goldzweig et al. (2009) per il periodo 2004-2007; Buntin et al. (2011) dal 2007 al 2010.
} 
hanno messo in discussione questi risultati sia per l'eccessiva brevità dell'orizzonte temporale considerato sia per l'eccessiva restrizione degli indicatori economici e sociali considerati. Infatti, data la varietà dei titolari di interesse coinvolti nei processi di e-health - pazienti, organizzazioni, professionisti, produttori, finanziatori e società nel suo insieme - all'analisi economica in senso stretto è spesso opportuno affiancare un'analisi di impatto sociale. Al riguardo, il progetto IMPACT (2006) della UE ha sviluppato un modello di valutazione costi-benefici che quantifica i benefici - diretti e indiretti - anche la stima della disponibilità a pagare per le innovazioni e mostra che sono mediamente necessari almeno quattro anni prima che i benefici superino i costi. ${ }^{11} \mathrm{Si}$ conferma quindi che il declino di produttività associato ai primi anni di adozione di innovazioni ICT è dovuto a una fase di adattamento che caratterizza tutte le tecnologie la cui efficacia dipende da una rilevante riconversione del capitale umano necessario per un loro efficace impiego. ${ }^{12}$ Superato questo periodo di riconversione, secondo Danzon e Furekava (2010), il maggior potenziale delle ICT nel migliorare l'efficienza dei sistemi, può essere conseguito proprio nel settore sanitario in virtù non solo della sua rilevanza $(9-17 \%$ del PIL) ma anche del suo essere particolarmente information-sensitive.

Con riferimento all'analisi delle aree specifiche in cui si osservano i principali effetti delle ICT, questi sono ad oggi ancora concentrati nei processi di supporto alla gestione dei processi amministrativi. Tuttavia, è prevedibile che con il crescente impiego di modelli previsionali in grado di assistere $i$ professionisti nei propri processi decisionali, in futuro assumeranno una importanza maggiore i miglioramenti direttamente riferibili ai costi e benefici dei processi assistenziali. ${ }^{13}$ Al riguardo, mentre

11 Questo studio si riferisce a dieci innovazioni suddivise tra Germania, Svezia, Romania, Francia, Repubblica Ceca, Belgio, Danimarca e Regno Unito. Un esempio di valutazione italiana che applica la stessa metodologia è il progetto ESCAPE (Electronic Signature in Care Activities for Paper Elimination») nella AUSL 9 di Treviso (Buccoliero et al. 2008).

${ }^{12}$ Davis (1989) ha definito tale fenomeno come "presbiopia tecnologica", nel senso che sono state sviluppate eccessive aspettative riguardanti l'impatto delle ICT in relazione al tempo occorrente perché gli effetti positivi si potessero determinare.

${ }^{13}$ I modelli predittivi di rischio clinico sono modelli che combinano un certo numero di caratteristiche (del paziente, della malattia, del trattamento) per fornire indicazioni in merito a diagnosi o prognosi mentre con il termine electronic health care predictive analytics (eHPA) si indicano gli applicativi le tecnologie in grado di dare applicazione autonoma ai modelli predittivi di rischio clinico. Lo sviluppo e l'implementazione di un e-HPA è costituito da quattro fasi: acquisizione dati per costruire il modello predittivo del rischio clinico, costruzione e validazione del modello; applicazione ad un ambito reale e applicazione del software, trasferimento del modello su ampia scala per applicarlo nel 
in altri comparti industriali, l'uso dei big data in chiave predittiva ha prodotto significativi miglioramenti di efficienza (Amazon ad esempio), la sua diffusione in ambito sanitario è ancora limitata, nonostante l'emergere di partnership tra alcuni finanziatori di servizi sanitari e grandi imprese del settore quali Google e Ibm, dedicate proprio all'impiego diretto di ICT nei processi di cura.

I dati disponibili sulla diffusione delle ICT in ambito sanitario, su cui si basano i lavori empirici sopra ricordati, provengono da rapporti istituzionali ricorrenti (Eurostat, Ocse, Istat) o da indagini specifiche. ${ }^{14}$ Secondo queste fonti, l'Italia sembra aver accumulato un ritardo rispetto ad altri paesi: in particolare il rapporto Desi 2015 della Commissione Europea, che valuta lo stato di avanzamento degli Stati membri verso un"'economia digitale", colloca l'Italia al $25^{\circ}$ posto nella classifica dei 28 stati membri della UE. Rispetto al dato medio nazionale, le Regioni che sembrano caratterizzarsi maggiormente per un diverso grado di «capacità digitale» sono l'EmiliaRomagna al primo posto e il Molise all'ultimo. ${ }^{15}$ Tuttavia, per buona parte delle innovazioni esaminate, $i$ dati disponibili non sono sufficienti a fornire una descrizione analitica della loro diffusione nelle strutture del Servizio sanitario nazionale, né a formulare valutazioni comparative di impatto, anche a causa dei diversi contesti organizzativi in cui si collocano le innovazioni. Oltre a queste difficoltà e alla conseguente scarsità di analisi empiriche, la letteratura nazionale non sembra convergere su un modello univoco di valutazione dell'impatto delle innovazioni ICT come quello che si sta imponendo nella letteratura internazionale, ${ }^{16}$ e che fa riferimento a

sistema sanitario. Il loro utilizzo in chiave predittiva potrà diffondersi più rapidamente in ambito ospedaliero ma successivi benefici potranno ottenersi in ambiti assistenziali diversi.

${ }_{14} \mathrm{Si}$ vedano, ad esempio, in ambito europeo, l'indagine "Health Consumer Powerhouse" o, per l'Italia, quelle predisposte da Ce.Ri.S.Ma.S, Confservizi, Confindustria, Federsanità-ANCI e ForumPA, Ministero Salute, ecc.

${ }^{15} \mathrm{Si}$ veda Telecom (2015). La Regione Emilia-Romagna, insieme alla Thomas Jefferson University ha lanciato nel 2014 un progetto diretto alle cure intermedie che prevede di sviluppare un modello predittivo per identificare i pazienti ad alto rischio di morte o di ospedalizzazione e attribuire ad ogni cittadino maggiorenne un «rischio ospedalizzazione» da comunicare alle case della salute (Louis et al., 2014).

16 Ad esempio, il Treasury Green Book nel Regno Unito (https://www.gov.uk/government/publications/the-green-book-appraisal-and-evaluation-incentral-governent\#history), gli studi della Rand Corporation e di Rand Europe (www.rand.og), dell'e-Government Economics Project (http://www.ehealth-impact.org/) e infine, dello studio e-Health Impact della Commissione Europea (https://ec.europa.eu/digital-single-market/en/european-egovernment-action-plan-20112015). 
tecniche di valutazione costi-benefici applicate a tutti gli elementi direttamente e indirettamente influenzati dalle stesse innovazioni.

L'opportunità di migliorare la qualità dei dati e soprattutto di potenziare le valutazioni d'impatto dell'introduzione di ICT nel Servizio sanitario è motivata soprattutto dalla necessità di definire una strategia complessiva in questo ambito da parte delle singole Regioni o del Governo nazionale. Al riguardo, un influente studio (Draca et al., 2006) ha da tempo posto al centro del dibattito il disegno ottimale delle politiche pubbliche a sostegno degli investimenti in ICT che devono necessariamente considerare anche gli investimenti nei principali fattori complementari a tali tecnologie che ne influenzano pesantemente gli esiti. ${ }^{17}$ All'interno del Servizio sanitario nazionale, il quadro delle politiche regionale di investimento in ICT è eterogeneo e sotto questo profilo va segnalato il recente Patto per la sanità digitale con il quale il Ministero della Salute si pone l'obiettivo di costituire una banca dati delle iniziative intraprese dalle regioni e dei relativi risultati e testimonia la consapevolezza della rilevanza del problema quando scrive che: «con la chiusura di presidi sanitari, la loro trasformazione, il potenziamento delle cure primarie e l'articolazione della rete ospedaliera in hub \& spoke, l'innovazione digitale può svolgere un ruolo chiave sia nell'evoluzione contemporanea dei modelli assistenziali, sia in quelli organizzativi, come fattore abilitante e in taluni casi determinante per la loro realizzazione». ${ }^{18} \mathrm{~A}$ fronte di questa consapevolezza, è importante che le strategie di investimento pubblico sulle ICT siano definite tenendo conto del più ampio contesto in cui esse si collocano. In particolare, occorre che esse prevedano adeguati piani formativi volti a superare la generale difficoltà di riconversione del capitale umano dei professionisti il cui apporto è essenziale per realizzare i benefici potenziali delle nuove tecnologie, nonché di tenere conto - soprattutto nel caso di innovazioni nel processo assistenziale - delle resistenze dei professionisti le cui funzioni sono direttamente modificate con l'adozione delle nuove tecnologie.

${ }^{17} \mathrm{Al}$ riguardo, negli Stati Uniti, per accelerare l'adozione di ICT in ambito sanitario, nel 2009 l'amministrazione Obama ha approvato l'American Recovery and Reinvestment Act (ARRA) che prevede un investimento di trenta miliardi di dollari per promuovere la diffusione delle tecnologie digitali in ambito medico. Fa parte di questo pacchetto normativo anche l'Health Information Technology for Economic and Clinical Health Act (HITECH) che introduce pagamenti ad incentivo per ospedali e professionisti che adottino tracciati elettronici e li usino nei percorsi di cura, contemplando nel contempo una riduzione dei finanziamenti pubblici per quanti, invece, optano di non farlo.

${ }^{18} \mathrm{http}: / / w w w . q u o t i d i a n o s a n i t a . i t / a l l e g a t i / a l l e g a t o 1787122 . p d f$ 


\section{Investimenti in ICT per la gestione integrata dei servizi sanitari}

In sezione 5 sono state analizzare le ragioni per cui, nonostante una generale tendenza contraria, in ambito sanitario la necessità di considerare il servizio fornito da un finanziatore come un insieme di attività integrate volte alla prevenzione e al recupero delle migliori condizioni di salute per gli assistiti, determina un vantaggio comparato per le organizzazioni che si integrano su tutte le fasi dei percorsi assistenziali. Questo vantaggio comparato, tuttavia, diviene effettivo solo se tali organizzazioni investono adeguatamente nella gestione delle infrastrutture e delle tecnologie necessarie per acquisire e valorizzare al meglio le informazioni necessarie a coordinare, valutare e riprogettare l'organizzazione dei servizi. Oltre al miglioramento complessivo nella qualità dell'offerta di servizi, vi sono ulteriori motivazioni specifiche che giustificano gli investimenti in ICT da parte delle organizzazioni che forniscono servizi sanitari secondo un modello integrato. La presente sezione è dedicata a una loro rapida analisi mirata a comprendere meglio le ragioni per cui tali organizzazioni - titolari dei diritti di uso delle informazioni - nel definire le proprie strategie in questo ambito devono tenere in considerazione quali sono gli incentivi degli altri soggetti che operano nel settore (erogatori di servizi, produttori di farmaci e di dispositivi biomedici, imprese specializzate in ICT, società di consulenza) interessati all'utilizzo degli stessi dati.

Per quel che riguarda i percorsi assistenziali, i più recenti sviluppi in tema di test genetici anche per l'impiego selettivo di farmaci specifici e il potenziamento delle tecnologie per la diagnostica per immagini, richiedono ingenti investimenti in infrastrutture per l'ordinata conservazione di una mole crescente di dati - su vari supporti e con varie modalità di raccolta -. In particolare la crescita esponenziale delle analisi sui profili genetici e più in generale su materiali biologici richiede un maggiore impegno nella costituzione di bio-banche accreditate per diverse tipologie di materiale, in grado di supportare la fase diagnostica e di personalizzazione dei percorsi assistenziali, ma necessarie anche per valorizzare appieno le attività di ricerca e di sperimentazione clinica. Allo stesso tempo, sempre con riferimento al supporto ai percorsi assistenziali, è opportuno investire nello sviluppo di dispositivi in grado di raccogliere informazioni in continuo sui parametri clinici di pazienti in fase acuta e ancor più di pazienti cronici in fase di stabilizzazione, riabilitazione o di mantenimento. Questi investimenti devono estendersi alle tecnologie complementari che consentono di seguire i pazienti in contesti domiciliari o in strutture a minor 
intensità d'uso delle risorse, mantenendo la possibilità di intervenire rapidamente in caso di peggioramento, come pre-condizione necessaria all'attuazione di percorsi assistenziali che non solo aumentino l'efficienza del sistema, ma consentano agli assistiti e ai loro familiari di migliorare la qualità di vita e ai finanziatori di programmare un impiego più appropriato degli investimenti strutturali.

Sul fronte dei processi gestionali, la crescente possibilità di raccogliere informazioni sui consumi sanitari in ogni fase e livello assistenziale, fino al singolo paziente - e in questo caso di poter disporre anche di dati da registri clinici -, consente di affinare gli strumenti di analisi per migliorare le attività di programmazione e di gestione. Al riguardo, a fronte delle stringenti necessità di bilancio, le organizzazioni sanitarie dovrebbero impegnarsi nell'analisi comparata di costo-efficacia di singoli trattamenti (ad es. farmaci ad alto costo, dispositivi biomedici o procedure chirurgiche), di interi percorsi assistenziali o di specifiche soluzioni organizzative. Sulla base dei risultati di tali analisi, potranno essere modificate le indicazioni di appropriatezza per tutti i professionisti e le strutture facenti capo all'organizzazione. Allo stesso tempo, più sul piano delle verifiche dell'uso efficiente delle risorse, sono ormai operativi sistemi di monitoraggio dei comportamenti prescrittivi di professionisti o delle caratteristiche dell'offerta dei singoli erogatori. ${ }^{19}$ Sempre sul piano gestionale, maggiori investimenti nella capacità di analisi delle informazioni sui consumi sanitari e sui dati clinici può consentire di dare applicazione a nuovi modelli di remunerazione di fattori produttivi ad alto costo. Infatti, a seguito della richiesta di numerose imprese farmaceutiche, avallata da diverse autorità di regolamentazione, che chiedono di essere remunerate per i farmaci innovativi in relazione al valore da essi creato in termini di benefici non solo di salute, ma anche in relazione alla riduzione prospettica dei costi per i finanziatori del sistema, questi ultimi devono essere siano in grado di effettuare proprie valutazioni sull'effettiva entità di questi risparmi. Inoltre, il crescente ricorso a percorsi integrati richiede di sperimentare forme di finanziamento dei singoli responsabili dei percorsi o delle singole strutture coinvolte che incentivino questi ultimi a effettuare interventi in grado di tenere sotto controllo $i$ costi complessivi del percorso,

\footnotetext{
${ }^{19}$ Non è casuale che a fianco delle società di consulenza più consolidate e specializzate nelle analisi di cui sopra, negli ultimi anni altre grandi imprese - non caratterizzate per una specializzazione in ambito sanitario - come Google o $\mathrm{Ibm}$ abbiano impegnato risorse ingenti sullo sviluppo di tecnologie a supporto delle attività di sperimentazione clinica, alla messa a punto di applicativi utili alla raccolta e alla condivisione di dati clinici e infine all'impiego di strumenti analitici per il supporto dei processi decisionali dei professionisti.
} 
senza scaricare su altri una maggiore quota di costi. Anche in questo caso, il disegno di forme di finanziamento che incentivino una gestione davvero integrata di un percorso assistenziale richiede investimenti non marginali nell'analisi dei dati rilevanti.

Infine, come per ogni altra organizzazione che, dopo l'avvento di internet, si interroga sulle valutazioni di qualità degli assistiti nei confronti della propria offerta di servizi, si pone l'opportunità di dotarsi di strumenti di analisi delle informazioni desumibili dai social network per comprendere gli elementi che condizionano gli assistiti nella loro scelta dei professionisti e delle strutture a cui rivolgersi e che ostacolano (o facilitano) le azioni volte a migliorare la prevenzione.

\section{Conclusioni}

In questo lavoro, partendo dall'approccio teorico della New Institutional Economics e più specificamente dalla teoria dell'impresa di Coase, sono stati analizzati gli effetti dei crescenti investimenti in ICT sui confini delle imprese, con particolare riferimento alla dimensione dell'integrazione verticale. Questa analisi è stata poi applicata in modo più specifico alle caratteristiche del settore sanitario nella complessa interazione tra finanziamento ed erogazione dei servizi che - a parità di altre condizioni motiva la prevalenza nell'adozione di modelli organizzativi verticalmente integrati o facenti leva su contratti relazionali.

L'analisi dell'impatto delle ICT sulle organizzazioni sanitarie fa emergere un effetto assai diverso dall'accorciamento dei legami verticali tra varie fasi della produzione che si osserva tendenzialmente in altri ambiti. Infatti, la crescente evoluzione dei servizi sanitari ad organizzarsi quali insieme di attività e servizi eterogenei ma fortemente integrati e finalizzati alla prevenzione, al mantenimento e al recupero del migliore stato di salute possibile, amplifica i vantaggi comparati derivanti da una gestione integrata anche dei flussi informativi che, a sua volta, rafforza i vantaggi di soluzioni organizzative verticalmente integrate.

In particolare, nel caso dei servizi sanitari, i flussi informativi interessati dalle grandi trasformazioni nelle ICT non riguardano solo le caratteristiche dei consumi sanitari e i loro esiti di breve termine, ma anche le condizioni di contesto - incluse l'evoluzione delle condizioni di salute dei singoli assistiti nel tempo - che determinano la scelta dei percorsi assistenziali di lungo termine e ne influenzano l'efficacia. Queste ultime caratteristiche sono invece di particolare rilevanza per una efficiente gestione dei percorsi 
assistenziali basata sulla capacità di controllare l'apporto dei diversi fattori produttivi impiegati, nonché per mettere a punto strumenti di valutazione dell'efficacia di singoli trattamenti, di percorsi assistenziali e di interi modelli organizzativi, necessaria a garantire la sostenibilità dei sistemi sanitari nel loro complesso.

Il lavoro si conclude con un esame della letteratura sull'impatto delle ICT sui livelli di efficienza nella gestione dei servizi sanitari e sulla qualità dei percorsi assistenziali. Tale analisi, mette in luce, tra le altre cose, l'importanza di considerare quali sono le condizioni che consentono di facilitare l'adozione delle innovazioni e di dispiegarne completamente gli effetti positivi, con particolare riferimento agli incentivi rivolti al personale sanitario che vuole tutelare il valore del proprio capitale umano non riducendo drasticamente le funzioni attribuitegli nello status quo. Queste considerazioni sono di rilievo nella definizione delle politiche pubbliche di sostegno alle ICT che devono inoltre considerare anche il ruolo cruciale di tali tecnologie per governare alcuni segmenti dei percorsi diagnostici, terapeutici e assistenziali (profili genetici, farmaci ad costo, diagnostica per immagini) legati alla gestione di volumi sempre crescenti di dati, per effettuare valutazioni comparative sui singoli trattamenti, così come sui modelli organizzativi, e per mettere a punto strumenti più sofisticati per il finanziamento dei servizi e la remunerazione delle strutture.

\section{Riferimenti bibliografici}

Buntin M.B., Burke MF., Hoaglin M.C. and Blumenthal D. (2011). The benefits of Health Information Technology: a review of the recent literature shows predominantly positive results. Health Affairs, 30 (3): 464-71, doi: 10.1377/hlthaff.2011.0178.

Blois K.J. (1972). Vertical Quasi-Integration, Journal of Industrial Economics, 20, 253-72, doi:10.2307/2098058.

Bresnahan, Timothy F., and Manuel Trajtenberg. (1995). General purpose technologies 'Engines of growth'? Journal of econometrics, 65.1: 83-108, doi: $10.3386 / \mathrm{w} 4148$.

Buccoliero L., Calciolari S., and Marsilio M. (2008). A methodological and operative framework for the evaluation of an e-health project. The International journal of health planning and management, 23.1: 3-20, doi: 10.1002/hpm.881.

Chaudhry B,Wang J,Wu S, Maglione M, Mojica W, Roth E, et al. (2006). Systematic review: impact of health information technology on quality, efficiency, and costs of medical care. Ann Intern Med,144 (10):742-52, doi:10.7326/00034819-144-10-200605160-00125. 
Coase R. (1937). The Nature of the Firm, Economica, 4; trad. it. La natura dell'impresa, in Impresa, mercato e diritto, Bologna, Il Mulino, 1995, 73-96.

Danzon, P.M., Furukawa, M. (2010). E-Health: effects of the Internet on competition and productivity in health care, in The economic payoff from the internet revolution, Litan, R E., and Rivlin A.M (eds). Brookings Institution Press.

Davis, F.D. (1989). Perceived Usefulness, Perceived Ease of Use, and User Acceptance of Information Technology. MIS Quarterly, 13, 3: 319-340, doi: $10.2307 / 249008$.

Dietrich M. (1994). The Economics of Quasi-Integration. Review of Political Economy, 6 (1), 1-18, doi: 10.1080/09538259400000001.

Draka M., Sadun R., Van Reenen J. (2006). Productivity and ICT: A Review of the Evidence. CEP Discussion Paper No 749, doi:10.1093/oxfordhb/9780199548798.003.0005.

Enthoven A. (1988). Theory and practice of managed competition in health care finance. Amsterdam: North Holland.

France G. (1998). Healthcare quasi markets in a decentralised system of government, in Bartlett W., Roberts LA, Le Grand J. (ed), Quasi-market reforms in the 1980s: a revolution in social policy, Bristol, The Policy Press.

Goldzweig CL, Towfigh A, Maglione M, Shekelle PG. (2009). Costs and benefits of health information technology: new trends from the literature. Health Affairs, 28(2):w282-93, doi: 10.1377/hlthaff.28.2.w282.

Grossman S., Hart O. (1986). The Costs and Benefits of Ownership: a Theory of Lateral and Vertical Integration. Journal of Political Economy, 94, 691-719, doi:10.1086/261404.

Hart O., Moore J. (1990). Property rights and the nature of the firm. Journal of Political Economy, 98, 1120-57, doi:10.1086/261729.

Light D.W. (2001). Comparative institutional response to economic policy managed competition and governmentality. Social Science \& Medicine, 52: 115166, doi: 10.1016/S0277-9536(00)00236-7.

Le Grand J. (2002). Further tales from the British National Health Service, Health Affairs, 21, 3: 116-129, doi: 10.1377/hlthaff.21.3.116.

Louis D.Z., Robeson M., McAna J. et al (2014). Predicting risk of hospitalisation or death: a retrospective population-based analysis. BMJ Open 2014; 4:e005223 doi:10.1136/bmjopen-2014-005223, doi:10.1136/bmjopen2014-005223.

Malone T., Yates J. e Benjamin R. (1987). Electronic Markets and Electronic Hierarchies. Communications of the ACM, 30: 484-497, doi $>10.1145 / 214762.214766$.

Odlyzko A. (2012). The volume and value of information. International Journal of Communication, 6: 920-935, doi:10.1126/science.1200970.

Solow R. (1987). We'd better watch out, New York Times Book Review, July 12 , page 36 .

Telecom Italia (2015) Italia connessa. Scaricabile online su http://italiaconnessa.telecomitalia.com/sites/default/files/ItaliaConnessa2015.pdf 
Ugolini C. (2004). Verso una rilettura del modello di concorrenza amministrata? Rivista delle politiche sociali, 4: 197-212.

Williamson O. (1975). Market and Hierarchies: Analysis and Antitrust Implication, Free Press, New York. 